\title{
Slobodan Jovanović et la tradition du libéralisme doctrinaire serbe : pour un changement $d u$ paradigme
}

Slobodan Jovanović and the Tradition of the Serbian "Doctrinaire" Liberalism

\section{Marko Božić}

\section{(2) OpenEdition}

\section{Journals}

Electronic version

URL: https://journals.openedition.org/ceb/5081

DOI: $10.4000 /$ ceb.5081

ISSN: 2261-4184

Publisher

INALCO

\section{Electronic reference}

Marko Božić, "Slobodan Jovanović et la tradition du libéralisme doctrinaire serbe : pour un

changement du paradigme", Cahiers balkaniques [Online], 42 | 2014, Online since 17 June 2014, connection on 07 July 2021. URL: http://journals.openedition.org/ceb/5081 ; DOI: https://doi.org/ 10.4000/ceb.5081

This text was automatically generated on 7 July 2021.

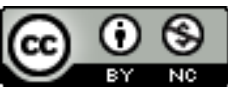

Cahiers balkaniques est mis à disposition selon les termes de la Licence Creative Commons Attribution - Pas d'Utilisation Commerciale 4.0 International. 


\section{Slobodan Jovanović et la tradition du libéralisme doctrinaire serbe : pour un changement du paradigme}

Slobodan Jovanović and the Tradition of the Serbian "Doctrinaire" Liberalism Marko Božić

\section{Introduction}

1 Si par paradigme on entend l'ensemble des croyances, des valeurs et des techniques partagées par une communauté scientifique déterminée (Khun, 1962), autrement dit l'idée dominante autour de laquelle gravite la pensée d'un milieu intellectuel et culturel et qui représente la cible privilégiée des critiques modernes et des théories alternatives, en ce cas, le paradigme de la théorie juridique serbe de la première moitié $\mathrm{du} \mathrm{Xx}^{\mathrm{e}}$ siècle est représenté par la pensée de Slobodan Jovanović et sa conception de l'État de droit. Sur ce point, la théorie juridique serbe reste unanime : pour la plupart des intellectuels d'hier et d'aujourd'hui, Jovanović a été peut-être un nationaliste convaincu et un théoricien conservateur du Staatslehre, mais surtout un fidèle sincère de la démocratie et du constitutionnalisme libéral. De son vivant, sa notoriété académique et sa figure patriarcale du droit public contribuaient déjà à la construction d'une légende autour de son œuvre. En outre, son destin malheureux lui a assuré le statut de victime du communisme, voire de martyre national ${ }^{1}$. Ainsi, aux louanges de ses contemporains s'ajoutent des panégyriques de la Serbie moderne qui le glorifie comme son saint profane. Hélas, la sainteté de son nom ne permet pas l'hérésie et peu d'auteurs ont essayé, ou mieux osé, mettre en évidence certains aspects controversés de sa pensée qui, bien qu'évidents, restent l'angle mort dans les analyses de son œuvre. Si autrefois la contestation idéologique des communistes interdisait un examen plus objectif, la science «libre » d'aujourd'hui ne fait pas mieux. Ainsi, bien qu'on dispose d'une bibliographie énorme dédiée à son œuvre entière, peu d'articles jettent la lumière sur les idées antithétiques de sa théorie juridique (Protić, 1998). Plus 
précisément, l'étrange mariage entre la théorie conservatrice de l'État de droit à l'allemande et le constitutionnalisme libéral à l'anglaise, semble laisser indifférents la plupart des auteurs contemporains. Personne ne semble s'étonner d'une conception de la démocratie qui refuse la souveraineté du peuple et cherche appui sur le modèle prussien de l'État fort et omniprésent.

2 Néanmoins, c'est bien cet aspect $a$-démocratique de Jovanović et de son libéralisme qui constituent le point crucial du paradigme serbe, ou ce qui rend sa pensée paradigmatique. En d'autres termes, même si l'on est d'accord sur le fait que Slobodan Jovanović a été « l'incontestable coryphée des intellectuels serbes de la première moitié du $\mathrm{xx}^{\mathrm{e}}$ siècle» (Petrović, 1998, 383) on ne le présente pas comme l'auteur de ce paradigme, mais on montre plutôt son œuvre comme sa meilleure expression. En effet, cette idée chimérique du libéralisme étatique ne dévoile pas seulement l'idéologie personnelle de Jovanović, mais aussi celle de sa génération et des générations suivantes, ou, si l'on veut, l'esprit national de la théorie juridique et politique serbe. D'ailleurs, on reconnait le reflet des mêmes idées dans la pensée des théoriciens conservateurs de l'époque, notamment celle de Živojin Perić, son homologue civiliste et l'un des ténors du Parti du progrès ${ }^{2}$. Cependant, ce qui assure l'identité serbe de ce paradigme n'est pas tant son originalité conceptuelle ni son ancrage profond dans l'esprit national, que le caractère endémique de sa mission politique ${ }^{3}$.

\section{Origine du paradigme, la méfiance à l'égard du peuple}

3 La fonction idéologique du paradigme serbe n'est pas évidente. Pour comprendre l'hybridation du libéralisme et de l'étatisme, il faut renverser la perspective: si le libéralisme serbe est un libéralisme étatique, c'est parce qu'il n'est pas démocratique. Chez les théoriciens serbes, l'accent est toujours sur l'État, jamais sur la société. Et, si la démocratie existe, c'est parce que l'État l'a instaurée par sa constitution écrite, le règne des lois et la séparation des pouvoirs. Autrement dit, l'État constitutionnel n'est pas la conséquence, mais la cause de la démocratie. C'est lui qui rend possible une société libre, et non l'inverse. C'est pourquoi si le paradigme serbe est libéral, il ne l'est guère dans le sens moderne du mot. Et si l'on peut parler de libéralisme serbe, ce n'est jamais celui qui nécessairement sous-entend la démocratie. En fait, dans cette mentalité, ce n'est pas la société civile qui engendre l'État, mais l'État qui précède et qui gère la société civile, où se cache sa véritable raison d'être.

\section{La base allemande - L'État de droit et le droit étatique}

4 Pour certains auteurs, la première édition de l'État de Jovanović n'est autre qu'un simple reflet de l'école de Staatsrecht et du soi-disant positivisme légal des auteurs classiques de la philosophie du droit allemande, Gerber, Laband et Jellinek (ibidem, 384). Ainsi, à la base, son système n'est que celui de l'école allemande de droit. En faveur d'une telle affirmation témoigne la reprise chez Jovanović des trois motifs cruciaux de ladite théorie germanique : la conception de l'État en tant que personne juridique, la distinction entre la loi au sens matériel et au sens formel, et enfin, la distinction entre les réglementations administratives et celles purement juridiques (ibid.). Sans la prétention d'élaborer ces trois points de coïncidence, on s'arrêtera sur l'examen de l'influence allemande sur sa conception de l'État de droit. 
5 Tous les auteurs serbes du début du xxe siècle soignaient le culte de la loi comme source unique, rationnelle et durable du droit. Ils l'accompagnaient du culte de la constitution écrite. Le respect de ce dernier est le résultat d'un lien prétendument logique entre deux concepts majeurs - celui du droit conçu comme la loi issue exclusivement de la volonté du législateur, et celui de la souveraineté en tant que propriété de l'organe législatif. En effet, à l'instar de Bodin et de la longue tradition de la pensée politique occidentale, dans la conscience politique serbe s'est cristallisée assez tôt la vieille idée de l'identité entre le pouvoir législatif et la souveraineté (Jovanović, 1991a, 146). Étant donné que la caractéristique principale de la souveraineté repose sur le pouvoir de création des normes générales, c'est-à-dire des lois, le corps législatif s'impose au moins comme le messager, sinon l'exécuteur, de la volonté souveraine, quel que soit son prétendu titulaire ${ }^{4}$. Le fait que l'État monopolise la souveraineté, et que cette dernière s'assimile au pouvoir législatif, la confection de la loi devient une compétence exclusivement étatique. Autrement dit, hors de l'État il n'existe pas de souveraineté, et par conséquent, pas de droit.

6 En suivant les pistes de la pensée allemande, Jovanović avance sur cette identité de l'État et du droit en précisant que « les organes législatifs exercent le pouvoir suprême dans un État, alors, le pouvoir souverain - et, en tant qu'exécuteurs du pouvoir souverain, ils représentent l'État à qui ce pouvoir appartient. L'État y trouve son image » (idem). Ainsi, chez Jovanović, comme chez ses maîtres allemands, disparaît la distinction entre le pouvoir législatif et la souveraineté, mais aussi disparaît toute possibilité - même hypothétique - de distinguer entre État et souveraineté conçue comme le pouvoir législatif. Autrement dit, dans le paradigme serbe, on ne voit plus de différence entre la souveraineté elle-même et son titulaire, ce qu'illustrent bien les propos de Živojin Perić qui déclare «S'il n'y a pas de puissance souveraine il n'y a pas d'État non plus: la souveraineté c'est l'État et l'État c'est la souveraineté » (Péritch, 1935, 233).

7 Ainsi, l'État est la personnification de la souveraineté, et cette dernière, à son tour, est l'unique productrice du droit sous la forme des lois. Il en résulte un lien ontologique entre l'État et le droit. Chez Jovanović, tout est identité. Et peu importe l'origine historique et le développement de cette unité. Ainsi, il propose

La meilleure option c'est de supposer que le droit et l'État sont nés en même temps que la vie sociale comme un ensemble inséparable. Le premier pouvoir jamais conçu représentait à la fois la première norme (...) et à l'inverse, la première norme jamais conçue représentait en soi un acte du pouvoir, car ceux qui l'ont créée devaient fonctionner en tant que titulaires du pouvoir au moment de sa création. Le pouvoir de l'État provient du droit, et le droit provient de l'État, et comme cela depuis l'origine, depuis que le pouvoir étatique et le droit sont apparus comme l'envers et l'endroit d'une même chose. (Jovanović, 1991a, 107) 5 .

Cependant, il faut noter que bien qu'il parle de l'État et du droit en tant qu'ensemble inséparable dont les deux éléments vont toujours de pair, il les distingue régulièrement, et l'on peut ainsi parler de deux entités distinctes. En fait, Jovanović ne soutient qu'en apparence la position moniste. Sa véritable position est dualiste, et sa mission idéologique est la justification de l'État par le droit: l'État n'apparaît plus comme la force pure, mais bien comme un État de droit, dont le fondement et la justification reposent sur le droit en même temps qu'ils le fondent ${ }^{6}$. C'est le concept de l'État autolimité?. 
Jusque-là, la théorie du droit étatique serbe, la mieux représentée dans la pensée de Slobodan Jovanović, suit fidèlement son modèle allemand. Le fait que Jovanović n'ait jamais accepté la prévalence de l'État sur le droit, mais qu'il prône une conception amalgame du droit et de l'État mystérieusement issus l'un de l'autre en même temps, ne diminue pas sa fidélité à ses maîtres allemands et ne représente pas, comme le prétendent certains, une originalité exceptionnelle de sa pensée ${ }^{8}$. Il n'est encore qu'un des métaphysiciens du Staatsrecht.

Jovanović semble prêt à faire un pas en avant quand il essaie de mettre cette théorie étatiste forte au service de la liberté individuelle. En effet, il se consacre à la création d'un concept hybride pour réconcilier ses deux convictions politiques de base - le besoin d'un État fort et la protection de l'homme en tant qu'être libre. En ce sens, Jovanović souligne que le problème privilégié de la théorie politique est depuis toujours celui de la réconciliation de la contrainte étatique et de la liberté individuelle (Basta, 1998, 346). C'est pourquoi il semble que la religion laïque de Slobodan Jovanović n'était pas simplement celle d'un État-dieu soumis à lui-même par l'intermédiaire du droit, mais plutôt celle d'un État auquel s'impose une mission supérieure : la protection de l'homme libre contre le despotisme. En ce sens, ce qui saute immédiatement aux yeux est son choix stratégique : au lieu d'avoir opté pour la démocratie comme la solution "naturelle ", il a embrassé une doctrine rigide, "prussienne », qui s'associe rarement aux démarches libérales. À première vue, on risque d'aggraver la confusion si l'on sépare de manière plus nette les mots de sa préférence et ceux de sa réticence: d'une part l'État et la liberté, de l'autre le despotisme et la démocratie. Cependant, c'est bien là le noyau axiologique et le point de départ idéologique de Slobodan Jovanović. Par conséquent, c'est sur cette idée chimérique que repose la vraie controverse de l'État de droit serbe et sa propre raison d'être.

\section{La greffe serbe, gouvernement du peuple, mais non par le peuple}

11 Il s'agissait, sans doute, d'un projet ambitieux : un État fort au service de la liberté individuelle, voire le vecteur principal de sa réalisation. On est loin d'affirmer l'originalité totale de Slobodan Jovanović sur ce point. D'ailleurs, il est clair que pour la théorie allemande, sa source d'inspiration et sa base spéculative, gouverner c'est commander les actions, mais toujours à des personnes libres. Il pourrait sembler que ces deux conceptions ne s'excluent pas tellement. Néanmoins, bien que Jellinek lui-même dise que le droit au sens politique n'est possible que parmi les hommes libres, pour les Allemands, le droit ne reste qu'un moyen rationnel pour l'accomplissement de la contrainte sur les hommes, libres, mais encadrés, tandis que selon la théorie libérale issue de la tradition britannique, le droit, et plus précisément la loi, est la meilleure garantie de la liberté individuelle. Tandis que le Staatsrecht allemand défend les sujets et leurs libertés en les limitant par la contrainte de la loi, le Rule of Law britannique part des individus libres qui recourent à la loi pour limiter l'interférence du pouvoir et protéger leurs libertés.

12 En fait, chez Jovanović, l'État de droit et les libertés individuelles forment un couple fusionnel, mais la justification de cette liaison n'est pas libérale. Jovanović ne croyait pas à l'origine naturelle des libertés de l'homme ni à la théorie du contrat social. L'État reste la véritable et unique origine des droits et libertés personnels. Toutefois, c'est bien la protection de ces droits et libertés qui assure le caractère moderne de l'État. En 
d'autres termes, il n'existe pas d'État de droit sans citoyens libres. Et pour deux raisons. « La première est utilitaire et fonctionnelle - seul un individu libre peut assurer à l'État moderne le niveau culturel d'où la communauté tire sa véritable force. La seconde raison réside dans le système juridique de l'état moderne qui exige que les citoyens soient des personnes égales en droits dont les libertés positives et négatives sont garanties par les droits publics subjectifs » (Pavković, 1998, 676). Ainsi, bien que chez Jovanović l'État ne représente pas le service de la Société civile, et qu'il existe indépendamment de l'individu, «il ne peut conserver cette existence collective, à laquelle il a autant droit que l'individu à son existence personnelle, que s'il élève ses sujets à un niveau culturel supérieur » (Jovanović, 1991a, 72).

13 Le trait particulier de la réinterprétation serbe de la théorie allemande du droit étatique, correspond donc exactement au paradoxe de son emploi émancipateur. Dans le milieu politique de la Serbie au tournant du siècle, elle a trouvé sa valeur pratique dans la promotion d'une sorte de régime que ses fondateurs allemands n'avaient pas particulièrement envisagé comme contexte concret de l'application de ses idéaux.

\section{Le mal du démocratisme}

Tout d'abord, il faut prendre en considération le fait que la théorie de Jovanović a été créée pendant «l'âge d'or » de la démocratie serbe, sous un régime politique né après le coup d'État de mai 1903 et le renversement du dernier roi de la dynastie Obrenović favorable à l'autoritarisme. Il s'agit d'un véritable coup de théâtre dans l'histoire moderne du pays, qui, pratiquement tout au long du xIX siècle, n'a connu que des régimes personnels de princes de moins en moins populaires. En Serbie, comme ailleurs, le combat de l'opposition libérale pour la réforme du cadre constitutionnel visait la limitation des pouvoirs quasi absolus des rois par la promotion des institutions parlementaires. La spécificité du combat serbe, parfois sanglant, réside dans le fait que l'opposition a trouvé son allié le plus loyal dans les masses paysannes, base du recrutement des électeurs du Parti radical, l'ennemi juré du régime. Leurs résultats furent mitigés et peu durables. C'est pourquoi le changement final de dynastie sur le trône serbe au début du $\mathrm{xx}^{\mathrm{e}}$ siècle est considéré par la conscience nationale comme une véritable entrée dans la modernité d'une nation depuis longtemps indépendante, mais politiquement peu émancipée.

15 La nouvelle constitution serbe de 1903 , en fait réplique de son précédent malchanceux de 1888, instaure une sorte de monarchie parlementaire, avec un rôle restreint du roi et une domination du gouvernement issu de l'Assemblée monocamérale. Cette dernière, à son tour, était élue en vertu du suffrage universel, ce qui donnait une image particulière de la Serbie de l'époque. En effet, il s'agissait alors d'un petit pays agraire, en marge des processus avancés d'industrialisation et d'urbanisation rapides en Europe occidentale. De là vint l'habitude de l'appeler « démocratie paysanne ».

16 C'est bien cette " démocratie paysanne » qui offre le cadre empirique aux réflexions de Jovanović, « libéral par son héritage familial, et conservateur par conviction politique » (Protić, 1998, 664). En fait, Jovanović s'est formé sous l'influence de deux courants distincts de la pensée politique : d'une part, cette foi de l'Aufklarung allemand en l'État, entendu comme ein Irdisch-Göttliches embrassant les particules individuelles dans sa supra-rationalité, d'autre part, les idéaux libéraux et la foi dans les capacités infinies de l'homme libre et indépendant. Cependant, s'il croyait à l'homme libre, il n'a jamais 
exprimé beaucoup d'engouement pour le peuple. Plus précisément, si les capacités infinies sont réservées aux hommes, pour Jovanović, ce sont toujours les individus particuliers qui en bénéficient, jamais les collectivités entières.

17 Ce détail de sa pensée devient évident dans ses analyses dédiées à l'État de droit. En effet, il est particulièrement important de noter que, chez Jovanović, l'identité de l'État et du droit a été régulièrement opposée à une autre théorie d'origine allemande, celle de l'école historique selon laquelle le droit n'est pas la décision rationnelle d'une autorité quelconque, mais, bien au contraire, l'émanation de l'esprit national dégagé spontanément dans l'histoire. Dans ses présentations de l'école historique, Jovanović rejette totalement cette affirmation forte selon laquelle le peuple seul est en capacité de créer le droit ${ }^{9}$. Il s'oppose au concept du droit donné sous forme de coutumes juridiques qui trouvent leurs sources dans une sorte de conscience populaire prétendument juridique. D'après lui, le meilleur motif pour la réfutation de cette théorie romantique est la prévalence évidente de l'État en tant que créateur exclusif du droit moderne. Alors, c'est bien la réalité empirique qui nous indique que le droit n'est point le produit d'une masse populaire inorganisée, ou le résultat d'une connaissance intuitive menée par les idées vagues de la justice. Bien au contraire, il ne peut être que le résultat d'un pouvoir législatif rationnellement organisé et le produit d'une longue délibération argumentée. Dans ces conditions, même si une coutume possède la force juridique, celle-ci est seulement due á son adoption par l'État et à sa transformation en loi (Jovanović, 1991a,56). Voire, "Le droit législatif est dans une bonne mesure étranger à la masse populaire. Une bonne partie de ses dispositions n'est compréhensible qu'aux classes instruites » (idem).

Cette opposition entre l'irrationalité populaire et la rationalité de l'État comporte-t-elle des implications politiques plus profondes? Une fois discrédité en tant que créateur fiable des normes juridiques, le peuple mérite-t-il une quelconque importance politique ? Plus précisément, l'État, a-t-il vraiment besoin du peuple à l'occasion de la production du droit? Si telle est la vraie question, on peut rester indifférent face aux vagues concepts métaphysiques du droit étatique de Jovanović. Car, cela étant, ce qui dévoile son orientation politique n'est pas tant ce que, d'après lui, le droit doit être, que, au contraire, ce qu'il ne devrait pas être.

19 À notre avis, et c'est la thèse que l'on pose ici, si les auteurs serbes ont recouru à la théorie allemande du Staatsrecht, c'est parce qu'ils n'avaient pas confiance en l'idée du Rule of Law dans sa forme originale. Autrement dit, la conception serbe du libéralisme au début $\mathrm{du} \mathrm{xx}^{\mathrm{e}}$ siècle, défendue dans la théorie du droit étatique de Jovanović, n'était pas du tout inspirée par le vieux concept anglo-américain d'un gouvernement du peuple par le peuple. Lui et ses semblables, comme Živojin Perić, ont reconnu le libéralisme plutôt comme une forme de parlementarisme issue de l'idéologie de la séparation des pouvoirs et des autres mécanismes préventifs du constitutionnalisme libéral, qui visent à empêcher la résurrection des régimes autoritaires. Alors, même s'il était un libéral, Jovanović ne l'était que dans un sens restreint, à cause de son opposition profonde au despotisme en tant que régime irrationnel et arbitraire, et non à cause de sa foi démocratique en la capacité autogestionnaire du peuple. D'ailleurs « Il est prouvé qu'il existe le despotisme du peuple, autant que le despotisme du prince » (Jovanović, 1897, 107), car "L'omnipuissance de l'Assemblée démocratique n'est pas moins négatrice à l'égard de la liberté individuelle que l'omnipuissance du prince " (Jovanović, 1991b, 153). Sur ce point, Živojin Perić est encore plus clair quand il dit que 
"L'absolutisme d'un seul est une maladie aiguë qu'on détecte sur-le-champ et pour cela on peut l'écarter. La démocratie ressemble à la maladie qui ronge l'organisme lentement et imperceptiblement" (cité par Popović-Obradović, 2008, 303). Par conséquent, l'absolutisme du prince est moins dangereux que le pouvoir illimité du peuple (idem).

Cette position de Jovanović et des conservateurs serbes n'étonne pas si l'on prend en considération le milieu social de son entourage. En effet, en Serbie de cette époque-là

... l'expérience des institutions du constitutionnalisme moderne était très modeste et la tradition parlementaire n'existe presque pas. En outre, dans la conscience politique dominante même le principe de la légalité n'a pas poussé les racines plus profondes. L'histoire de l'État et du droit de la Serbie du XIX siècle a été déterminée avant tout par les règnes personnels, les changements violents sur le trône, l'influence politique de l'armée, les révisions des constitutions par voie inconstitutionnelle, mais aussi par une influence forte des idéologies révolutionnaires, et particulièrement par cette tendance de concevoir la démocratie comme le règne absolu de la majorité. L'une des idées politiques les plus ancrées, celle qui présentait la pierre angulaire du programme constitutionnel de tous les mouvements de la réforme politique en Serbie durant le XIXe siècle [...] était bien l'idée du pouvoir souverain de l'Assemblée nationale. Par contre, c'était l'idéologie du libéralisme politique - l'idéologie sur laquelle ont été fondées les institutions du constitutionnalisme européen moderne - dont la racine était la plus faible. Le premier essai de l'introduction d'un régime de la démocratie parlementaire, fait par la Constitution de 1888, a subi l'échec précisément à cause $\mathrm{du}$ conflit entre deux tendances également antilibérales: d'une part, la préservation du pouvoir personnel du prince et, d'autre part, la compréhension du principe de la souveraineté du peuple comme le pouvoir de la majorité sans limites » (Popović-Obradović, 1998, 570).

21 À notre avis, pour Jovanović, le problème ne résidait pas tant dans le pouvoir illimité de l'Assemblée nationale en tant que représentante de la majorité parlementaire, que dans la menace du pouvoir absolu de la majorité tout court, c'est-à-dire le pouvoir des plus nombreux - la masse.

Il faut remarquer que, chez Jovanović, cette crainte vis-à-vis du pouvoir politique des masses va régulièrement de pair avec son élitisme. Parfois exprimé plus ouvertement, cet élitisme reste le plus souvent latent, mais toujours présent dans ses écrits.

La naissance de la démocratie parlementaire dans la Serbie du début du $\mathrm{xx}^{\mathrm{e}}$ siècle petit pays de paysans pauvres - aurait pu seulement augmenter cette peur déjà bien présente dans l'esprit et l'œuvre de Jovanović ${ }^{10}$. En ce sens, selon lui, le populisme militant et les idées radicales ne peuvent trouver meilleur terrain pour s'implanter que celui offert par une société agraire à la modeste culture démocratique. C'est pourquoi, bien que formé en tant que personne durant l'époque marquée par la lutte libérale pour la démocratie et le parlementarisme, il restait prudent face à la transformation politique de la Serbie de son temps. «Il ne fut jamais membre d'aucun parti politique et s'en tint toujours à distance. Il n'aimait pas ceux qui cherchaient le soutien dans les chiffres. Il a plutôt fait confiance à l'intelligence, qu'à la masse. Intimement, il reconnaissait la popularité et la puissance des radicaux, mais il les considérait comme démagogues et partisans... » (Protić, 1998, 663).

24 Selon Jovanović, la démocratie même est problématique, car elle s'appuie sur un soutien douteux, c'est-à-dire l'opinion des masses et « ... la masse n'a pas de sang-froid et elle n'est pas capable de tirer des conclusions mûres, elle s'incline devant ses 
impressions instantanées, elle est l'esclave de ses passions et ses faiblesses..." (Jovanović, 1991b, 799). De cette manière, il s'apparente aux intellectuels serbes proches du Parti du progrès. Ces derniers préfèrent parler de démocratisme que de démocratie, et déclarent que "L'erreur démocratique principale est précisément le gouvernement de l'État par le peuple. Le peuple ne peut pas gouverner l'État, car il n'a pas de sens pour le gouvernement étant donné qu'il ne sait pas distinguer les besoins réels. Au contraire, il les confond avec ses sympathies et antipathies instantanées " (Perić, 1910, 180). Forcément, la situation est pire dans les pays dont le peuple n'est pas suffisamment éclairé. Là, le gouvernement dépend exclusivement de la foule, et c'est pourquoi il ne peut pas travailler comme il le voudrait sur le progrès national. La masse ne peut pas tout simplement comprendre ni autoriser la réalisation d'idées monumentales (Čekić, 1908, 74). Dans un tel milieu social, la démocratie devient un frein au développement. C'est pourquoi leur discours sur la démocratie pourrait paraître frappant. Ainsi, selon eux, la démocratie n'apporte que « la désorganisation et l'anarchie " et s'assimile à "la politique de vacarme» et à «la maladie d'engueulement » (Stojanović, 2003, 143). En outre, les progressistes, avec le président de leur parti Stojan Novaković en tête, insistent régulièrement sur le fait que la tendance révolutionnaire est intrinsèque à la démocratie (Novaković, 1908). D'après Živojin Perić, les démocrates qui adulent le peuple jouent un jeu dangereux, et il ne faudra pas s'étonner s'il arrive un jour où le peuple demandera «la preuve de sa souveraineté plus réelle que le bulletin de vote " (Perić, 1910, 181). Voire, "dans son sens exact, le gouvernement du peuple et la Terreur sont identiques » (idem).

Pourtant, ce choix de vocabulaire dévoile bien plus qu'une simple réticence vis-à-vis de la démocratie. Si l'on sait que les progressistes soignaient normalement le ton modéré et raffiné de leur discours, on pourrait supposer que leur attaque frontale contre la démocratie porte une signification plus profonde :

Ils n'étaient pas d'accord avec les autres aspects du gouvernement radical non plus, mais ils ne se permettaient jamais d'abandonner la forme tolérante de leur communication politique qu'ils considèrent essentielle. C'est pourquoi on conclut qu'ils ont pris l'idée de la démocratie comme le symbole-clef du nouveau gouvernement. Par conséquent, pour mieux exprimer son attitude, ils ont frappé ce symbole avec les arguments plus forts (Stojanović, 2003, 147).

D'ailleurs, ils se sont identifiés en tant que conservateurs éclairés justement pour accentuer leur opposition par rapport à la démocratie (Novaković, 1908, 6). Néanmoins, ce conservatisme ne partait pas seulement du rejet de la démocratie à cause de son fondement populaire, mais aussi d'une foi en l'individualité exceptionnelle comme véritable porteuse du progrès. L'avancement de la civilisation est possible grâce aux idées des individus moralement et intellectuellement supérieurs aux foules. Naturellement, c'est à eux de gouverner (Čekić, 1908, 74). Dans cette perspective, le conservatisme traite le peuple comme un mineur qui nécessite un tuteur pour s'occuper de ses droits et de ses affaires (idem). En ce sens, un bref passage de Živojin Perić, où l'auteur plaide en faveur d'un parlement dressé au-dessus des masses populaires, est fort illustratif :

... le législateur ne se laisse pas conduire seulement par l'évolution du Droit telle qu'elle se dessine dans le peuple, mais il la précède très souvent en dotant le peuple des institutions qui n'y ont pas encore vu le jour, c'est-à-dire des institutions que sa raison construit d'une façon complètement spéculative. Ainsi, il parvient à faire progresser le Droit plus vite que ce ne serait le cas, s'il ne s'en remettait à cet égard qu'au lent développement de la conscience juridique populaire (l'évolution). Et, 
d'une manière générale, depuis toujours l'évolution culturelle de l'Humanité a été spécialement secondée et accélérée par des individualités dont l'esprit supérieur voyait, comme la vigie du bateau, plus loin dans l'avenir que la masse du peuple. Leur esprit transformait la société dans une espèce de serre chaude où tout grandissait et mûrissait plus vite. Ce sont surtout les individualités qui montraient et qui montrent à la société et à l'Humanité des nouvelles voies et des nouveaux horizons en élevant le genre humain au-dessus d'autres êtres de la Nature qui, dépourvus de la raison, sont soumis aux lois physiques et à l'évolution que ces dernières leur imposent " (Péritch, 1935, 238). Par conséquent, la division de la société en deux groupes selon leur degré intellectuel n'est pas tragique, car «ne peut pas avancer une société dont les groupes seraient tous au même niveau culturel. (Jovanović, 1991c, 255).

C'est une interprétation de la société très proche de celle de Pareto : tout simplement, certaines qualités se trouvent chez certains hommes et non chez d'autres. Cette inégalité est fondatrice et essentielle, car elle joue le rôle de dynamique même du tissu social. De ce point de vue, l'obsession d'égalité de la démocratie porte une menace sérieuse pour le fondement de la société, car elle a tendance à supprimer toute possibilité d'extraction naturelle des esprits extraordinaires (Čekić, 1908, 73). Et c'est la véritable peur de l'intelligentsia serbe - l'individualité perdue et noyée dans les profondeurs de «la mer agraire» de leur patrie. Cependant, il ne s'agit pas d'un phénomène spécialement serbe. Selon certaines opinions, c'est également une problématique importée de l'Occident. On sait que depuis son avènement, la démocratie suscitait chez certains libéraux, y compris Tocqueville, la crainte de voir les hommes politiques remarquables devenir moins remarqués et reconnus par les citoyens chargés de les porter au pouvoir par l'élection libre. Cette crainte de la médiocratie est justifiée par le suffrage universel et le présupposé selon lequel les gens ordinaires pourraient préférer des personnes leur ressemblant davantage. Il ne faut pas négliger le fait que

... tous les libéraux portent en premier lieu le regard sur l'individu dans sa singularité. Aussi n'est-il pas si étonnant qu'ils aient souvent une certaine difficulté à se représenter la démocratie, qui se fonde précisément sur une conception des hommes dans ce qui les rassemble, dans ce qu'ils ont de semblable. Très schématiquement, on pourrait ainsi dire de la démocratie qu'elle est une pensée de la société, du vivre-ensemble, le libéralisme se concevant davantage comme une pensée de la séparation, de la dissociation. (Leboyer, 2012, 114).

De manière dialectique, c'est bien cette dissociation qui offre le fondement de la cohésion sociale, car

décrire une élite politique, cela ne revient pas seulement à poser une distinction, celle que l'on trace entre les gouvernants et les gouvernés, mais également à déterminer ce qui relie ensemble les gouvernants comme les gouvernés. La distinction entre représentants et représentés ne peut en effet être conçue que si chacune de ces deux sphères est comprise dans sa singularité. En d'autres termes, penser la distinction implique de penser une certaine cohésion, permettant de saisir la place « des» gouvernants et « des» gouvernés (ibidem).

29 Au final, il semble que ce libéralisme élitiste n'est point une invention serbe, mais un phénomène inhérent au libéralisme. Selon certains auteurs, sa présence dans la théorie politique serbe est d'origine française et trouve son lointain prédécesseur dans la pensée politique de François Guizot et des doctrinaires français (Bataković, 1997, 83 ; Protić, 1990, 85). Plus précisément, il s'agit de la doctrine de l'aristocratie de la vérité formulée par Guizot dans Des moyens de gouvernement et d'opposition dans l'état actuel de la France de 1821. L'aristocratie de la vérité n'a rien à voir avec l'aristocratie caste du 
système de l'Ancien Régime. En réalité, sous cette notion, Guizot entend l'élite sociale composée des esprits supérieurs, et donc prédestinée à gouverner. Tandis que l'aristocratie de l'Ancien Régime était un phénomène artificiel, l'aristocratie véritable est un phénomène naturel dont la supériorité ne s'impose pas, mais émerge spontanément et se reconnaît facilement par l'intelligence, le courage, la justice, mais aussi le raffinement du goût de ses composants. C'est dans ce sens qu'il faut comprendre l'aristocratisme comme le principe de gouvernement prôné par les conservateurs serbes, autant que dans les propos de Perić quand il avertit que «le développement $d u$ démocratisme entraîne l'élargissement $d u$ suffrage et l'augmentation des éléments antiaristocratiques au sein du Parlement». En effet, même s'il a pensé à l'aristocratisme de sang, il a également parlé de celui fondé "sur l'éducation et les sentiments ou sur le raffinement du goût » (cité par Stojanović, 2003, 142).

En réalité, à défaut de l'aristocratie de sang, les conservateurs serbes ont été forcés de considérer l'intelligentsia nationale, celle du « raffinement du goût » comme l'unique possible « l'aristocratie de la vérité » serbe. Sa supériorité se reconnaît exactement par son aliénation de la base sociale. En effet, selon Jovanović, par intelligence nationale, on comprend la minorité éduquée et émancipée des masses paysannes, celle qui mène une vie culturellement plus sophistiquée. Cette minorité « séparée des masses paysannes par son mode de vie, elle a forcément dû se séparer de son mode de pensée aussi ; elle a acquis une vision du monde particulière - et il n'y a rien plus naturelle que le peuple ne la comprend pas et qu'il ne lui fait plus confiance » (Jovanović, 1991c, 255). D'ailleurs, ce sont bien cette aliénation et cette autosuffisance qui font l'élite reconnaissable (Leboyer, 2012, 99).

La conception d'une élite intransigeante, irréductible, retranchée dans ses pensées, met l'accent sur ce qui la distingue et l'isole. Ne pas écouter est ici le signe que l'on n'entend pas être dérangé par des considérations ou intérêts autres que les principes sur lesquels se règle de lui-même l'esprit [...] Elle ne saurait se laisser dicter sa conduite, et ce n'est jamais des autres que lui viennent ses intuitions [...] L'élite trouve en elle la force, les ressources et l'intelligence nécessaires pour prendre ses décisions, sans être influencée d'aucune sorte (Ibidem, 98).

31 Cependant, si cette confiance en soi se traduit par une autosuffisance, elle n'entend aucun autisme social. Dès, alors, l'élite n'est plus conçue seulement comme un groupe fermé, lointain et incompréhensible, mais d'abord, comme une classe sociale à part, celle des gouvernants.

\section{Le remède de l'aristocratisme}

Empêcher les masses illettrées, manipulées par leurs représentants politiques, de prendre les rênes du pouvoir devient également le premier souci de la pensée politique de Slobodan Jovanovići ${ }^{11}$. S'il était libéral, Jovanović l'était dans un sens faible, c'est-àdire en tant qu'élitiste, et c'est pourquoi il paraît juste de qualifier son concept de libéralisme avec réserve, en effet, en tant qu'élitiste. En effet, s'il admettait que le rôle de meneur du peuple devrait appartenir à ceux élus par le peuple lui-même, il se demandait aussi comment faire si ce peuple n'est pas suffisamment mûr pour faire le meilleur choix. C'est pourquoi «Dans les milieux politiquement retardés, comme l'était la Serbie aux yeux de Jovanović, il est nécessaire de trouver l'équilibre entre la démocratie et le gouvernement compétent » (Protić, 1997, 664). Tout simplement, il n'était pas persuadé qu'on peut efficacement gouverner un pays sous-développé en 
s'appuyant seulement sur la volonté d'un peuple non émancipé et composé de paysans. À l'instar du Parti du progrès, il a tenu à ce qu'en Serbie le régime parlementaire soit corrigé ou atténué par des institutions qui amélioreraient le niveau de l'éducation politique nécessaire parmi les représentants du pouvoir (idem).

Le point de départ de cette démarche était la disqualification de la base même de la démocratie : la souveraineté du peuple. Dans cette démarche, Jovanović n'était pas seul. En fait, ici aussi, il rejoint la longue lignée des conservateurs regroupés autour du Parti du progrès, et leur conviction commune que le despotisme apparaît chaque fois que le pouvoir législatif - la propriété cruciale de la souveraineté - est concentré aux mains d'un seul organe (Jovanović, 1991c, 254). En s'appuyant sur la doctrine de la séparation des pouvoirs, ils ont affirmé que du seul fait que la souveraineté est par définitionem indivisible ne découle absolument pas l'idée qu'elle doit être focalisée sur un même organe (Jovanović, 1991a, 131). Simplement, on n'a aucune raison de croire que les mains de l'Assemblée nationale sont moins avides que celles du Prince absolu. Voire

le souverain autocrate est, dans une certaine mesure, retenu dans ses actions par la crainte de la responsabilité, tandis que cela n'a pas lieu pour la représentation nationale qui s'est emparée de tous les pouvoirs: la représentation nationale n'étant qu'une foule, élue il est vrai, et la foule n'étant pas un être simple, unitaire, il n'est pas possible que la représentation nationale ait conscience de sa responsabilité - comme c'est le cas d'un individu pris isolément. (Péritch, 1904, 15).

C'est pourquoi considérer la majorité parlementaire comme unique vecteur de la volonté générale serait absurde. D'après Jovanović il s'agit d'un concept clairement antilibéral, car « La liberté est la limite de tout pouvoir ; pour qu'elle puisse exister, le pouvoir doit céder, et c'est pourquoi il n'y a pas de place pour la liberté à côté du pouvoir absolu même si ce pouvoir est exercé directement par le peuple lui-même » (Jovanović, 1897, 107). Au lieu de forcer une idéologie aussi erronée et dangereuse que la souveraineté de la Assemblée nationale, il vaudrait mieux assumer « qu'au contraire, dans la monarchie constitutionnelle moderne, le pouvoir législatif est partagée entre le monarque et le parlement, et le parlement est presque toujours divisé en deux chambres » (Jovanović, 1991a, 131).

Par conséquent, le côté élitiste du constitutionnalisme parlementaire de Jovanović et des théoriciens conservateurs du libéralisme-doctrinaire à la serbe ${ }^{12}$, se manifeste le plus clairement sur deux points : d'une part, sa conviction que la couronne représente le meilleur contrepoids à la majorité parlementaire dans le jeu législatif, et de l'autre, son plaidoyer pour une composition bicamérale du parlement.

\section{Le roi actif}

Si Jovanović était très loin de concevoir le régime démocratique comme le règne absolu de la majorité parlementaire, c'est parce que sa conception de la démocratie correspond bien plus aux idéaux du libéralisme européen classique du XIX ${ }^{e}$ siècle. Ce dernier n'excluait pas les éléments aristocratiques et élitistes comme le suffrage censitaire, la chambre des pairs et un rôle constitutionnel réservé à la couronne. C'est là l'origine du respect particulier que Jovanović portait à la couronne, considérée non seulement comme le chef nominal de l'État, mais aussi comme un facteur politique d'importance (Protić, 1998, 663).

En fait, son modèle d'un État moderne était celui de la monarchie constitutionnelle et parlementaire, fondée sur la séparation et l'équilibre des pouvoirs entre le roi et le 
parlement (Popović-Obradović, 1998, 571). D’après lui, le paradigme de la constitution libérale était représenté par la constitution belge de 1831, qui a envisagé un rôle politique très actif de la couronne comme le contrepoids du pouvoir de l'Assemblée nationale avec laquelle le roi partage le pouvoir législatif.

Le principe de Montesquieu selon lequel on ne peut laisser nul pouvoir sans limites est devenu le fondement de l'organisation étatique moderne. On évite l'omnipotence de l'Assemblée autant que l'omnipotence du prince... On a trouvé là le juste milieu entre ces deux despotismes dans le gouvernement parlementaire. Le prince et l'Assemblée se limitent respectivement... en gardant l'équilibre (Idem).

D'ailleurs, c'était un des points principaux du programme des progressistes, qui croyaient que la souveraineté n'appartient ni au roi ni au peuple, mais qu'elle est partagée entre ces deux facteurs constitutionnels égaux (Perić, 1908,24). Plus précisément, cet équilibre est le meilleur garant des libertés individuelles, car « Il n'y a pas de liberté là où il y a omnipuissance du prince ou celle de l'assemblée, au contraire, quand l'omnipuissance réside sur quelque chose qui ne s'identifie pas avec le prince ni avec l'assemblée, la liberté existe » (Idem, 92).

D'où le besoin d'un contrepoids à l'Assemblée nationale représenté par la couronne. De plus, chez Jovanović, la justification de la séparation des pouvoirs dépasse celle offerte par le libéralisme classique. En effet, à ses yeux, la couronne est nécessaire comme facteur correctif à la dangereuse influence du pouvoir absolu des masses. L'équilibre est possible, car, dans un système de monarchie parlementaire, aucune motion ne peut devenir loi si elle ne récolte pas le soutien des deux facteurs constitutionnels à la fois. En ce sens comme le frein particulièrement important de ce mécanisme du contrôle royal, il reconnaît le droit à la couronne de dissoudre l'Assemblée, qui, en revanche, n'a aucun droit réciproque vis-à-vis de la durée du pouvoir royal.

Ici, il est important de noter que par ces réflexions, Jovanović n'a fait qu'offrir sa contribution à la théorie du roi actif, bien plus développée dans l'œuvre de Živojin Perić. Il s'agissait d'une doctrine allant à l'encontre de la position du Parti radical au pouvoir, selon laquelle une monarchie parlementaire entend le rôle passif du roi, conseiller qui laisse l'Assemblée démocratiquement élue mener librement l'État. Partant de l'idée que l'autorité est synonyme de fonction, c'est-à-dire une compétence qui doit être exercée, Perić conclut que "Par conséquent, le monarque est tenu, de par la Constitution, d'exercer ses fonctions ; en ne les exerçant pas, il néglige les devoirs que la Constitution lui a imposés... » (Péritch, 1904, 9). Autrement dit, le monarque constitutionnel est obligé d'exercer ses prérogatives envisagées par la Constitution. Ainsi, outre l'inconstitutionnalité active, qui correspond à l'action qui outrepasse ses limites constitutionnelles, le roi peut également commettre une sorte de l'inconstitutionnalité passive, « celle qui consiste dans le non-accomplissement, de sa part, des fonctions que la loi fondamentale de l'État a placées dans ses attributions. Ces fonctions, en effet, ne constituent pas les droits du monarque, mais ses devoirs: ce qui veut dire qu'il ne dépend pas de sa volonté de les exercer ou non » (idem, 8).

41 C'est bien cette dernière constatation qui distingue les positions relatives de Perić et de Jovanović. D'après ce dernier, l'approche de Perić est restée exclusivement juridique. En se limitant à une analyse strictement normative, il a rendu sa théorie trop rigide et sèche. Effectivement, il a oublié que la Constitution ne représente qu'un cadre formel du combat politique et que, par conséquent, le rôle du roi ne dépend pas tellement de ses prérogatives constitutionnelles, mais plutôt de sa force politique réelle. Étant donné qu'il s'agit ici d'un jeu de forces, selon Jovanović, même le non-accomplissement des 
fonctions royales est un acte dont la motivation, la connotation et les résultats sont purement politiques (Jovanović, 1938, 6). De ce fait, la thèse sur l'inconstitutionnalité passive n'est pas pertinente. C'est probablement ce sens de la realpolitik qui a mené Jovanović à changer sa propre stratégie. Alors, bien qu'il ait continué à s'opposer à la théorie et à la pratique de la souveraineté de l'Assemblée nationale, il a entre-temps abandonné sa position initiale sur un rôle politique fort du roi. Au fil du temps, avec le renforcement des idées démocratiques, il devenait de plus en plus difficile de maintenir l'équilibre entre le parlement et le prince : la volonté du peuple, exprimée à l'élection, était de plus en plus souvent considérée comme la volonté supérieure, sans parité avec aucune autre volonté. Y compris celle du prince lui-même (Protić, 1998, 663).

Ainsi, en adoptant la position de Thiers selon laquelle « Le roi règne, mais ne gouverne pas", Jovanović s'est tourné vers la deuxième ligne de défense de son système antipopuliste - la composition bicamérale du parlement ${ }^{13}$.

\section{Le bicaméralisme}

Il est important noter que l'apologie théorique de cette idée a connu une évolution. Au début, la chambre haute était considérée comme un facteur d'équilibre, en effet, une institution intermédiaire, presque médiatrice entre le roi et l'Assemblée nationale. Elle rend possible l'équilibre des pouvoirs et, par conséquent, constitue comme la partie régulière du mécanisme complexe du régime parlementaire (Jovanović, 1991c, 269). Jugée indisponible, son introduction offre une possibilité d'affirmation politique à l'intelligence conservatrice. En effet, malgré leurs conceptions différentes à l'égard de sa composition et de son éligibilité - la préférence pour la nomination ou l'élection de ses membres, par exemple -, les partisans du bicaméralisme étaient unanimes sur un point : la chambre haute serait composée des représentants de l'intelligence nationale. C'est pourquoi ils l'ont crue capable non seulement de s'imposer contre les ambitions despotiques respectives du prince et du peuple, mais aussi de reconnaitre au mieux l'intérêt public et de remplir sa tâche législative (Perić, 1903). Avec l'instauration du système parlementaire par la nouvelle Constitution de 1903, la perspective change et l'idée de la chambre haute devient un argument des adversaires de la démocratie populaire. À présent, on la justifie comme le facteur correctif, posé à l'encontre de l'Assemblée, dont le niveau intellectuel est douteux. Alors, au premier plan, apparait sa nature élitiste. Si elle reste un instrument d'équilibre, l'accent ne porte plus sur l'idée de balance, mais plutôt de contrepoids ${ }^{14}$.

On rencontre la même transformation de l'usage politique de cette idée dans l'œuvre de Slobodan Jovanović. En témoigne le remaniement de sa défense doctrinaire du bicaméralisme, qui, après 1903, vise exclusivement le quasi-despotisme de l'Assemblée nationale. Certes, pour les partisans du système monocaméral, qui ont défendu le pouvoir souverain de l'Assemblée nationale, l'introduction du sénat aurait constitué une mesure portée directement à l'encontre de l'idée de démocratie. Ainsi, pour Gligorije Geršić, principal idéologue du Parti radical, le sénat est un résidu du féodalisme et de l'aristocratisme, ce que Jovanović n'a d'ailleurs jamais nié ${ }^{15}$. Pour Geršić, le sénat contredit le principe élémentaire de la démocratie conçue selon le raisonnement de Sieyès, pour qui la loi est le produit de la volonté populaire, et étant donné qu'un peuple ne peut avoir deux volontés différentes sur le même objet en même temps, il en résulte que la souveraineté populaire ne peut être organisée que sous forme d'un parlement monocaméral (Jovanović, 1932b, 28). Le contre-argument 
principal de Jovanović était qu'une majorité à l'Assemblée nationale, issue d'une élection libre, ne représente jamais l'ensemble de la nation et, par conséquent, ne peut jamais exprimer la volonté pour la nation entière. Face à la majorité issue de l'élection, il existe également une minorité qui ne parvient pas à s'imposer et à envoyer ses représentants au parlement « dominé par les gens de la campagne et des bourgs, avec les horizons étriqués » (Jovanović, 1991c, 270).

D'ailleurs, cette minorité ne trouve pas sa légitimité dans le nombre, mais dans son instruction et sa position sociale. D'après Jovanović la présence de l'élite dans le travail de l'organe législatif est nécessaire en tant qu'élément politique dont le rôle est de corriger les fautes des représentants du peuple réunis dans l'Assemblée nationale. Cependant, ce rôle de contrôleur n'est pas jouable si les représentants du peuple et de l'élite sociale se confondent dans le cadre d'un même corps législatif, où les premiers l'emporteraient facilement sur les seconds dû à la loi du nombre : « ... quelle erreur de penser que les éléments qui doivent empêcher les fautes de la représentation populaire, la contrôler et lui résister, pourraient vraiment en faire s'ils étaient eux-mêmes les membres du même organe" (Slobodan Jovanović, 1932b, 41). Ici plus que jamais, Jovanović montre son visage de conservateur qui ne croit pas trop à la souveraineté populaire dont l'expression est l'Assemblée nationale élue librement en vertu du suffrage universel.

Dans les Assemblées nationales serbes - expliquait Jovanović - c'est la couche des paysans qui domine et qui sans frein représentée dans le sénat, primo, ne peut pas garantir la compétence et la responsabilité adéquate de la législation et, secundo, par son attachement à l'idée de la souveraineté du peuple menace le principe de l'équilibre des pouvoirs en essayant de lui opposer la volonté souveraine de la majorité. (Popović-Obradović, 1998, 572).

\section{Justification du paradigme - la neutralité de l'État}

D'après Jovanović la société serbe du XIXe siècle n'était tout simplement pas assez mûre ni consciente, et sans la capacité autonome de se conduire seule et de reconnaître spontanément les préceptes de son autodétermination. À ses yeux, la démocratie libérale moderne apparaît comme un projet trop ambitieux pour un petit pays balkanique sans expérience ni culture politique. C'est pourquoi cet intellectuel élevé dans une tradition libérale, intellectuellement formé à l'Occident et théoriquement intéressé par la protection des droits individuels, a préféré une approche tempérée, voire conservatrice. La démocratie n'est pas son idéal, mais plutôt une idée-force remarquée (Stanovčić, 1998, 646). Son idéal est autre, c'est l'État fondé sur la loi, l'État de droit.

En fait, si la société serbe n'était pas encore prête pour une autorégulation, l'État serbe méritait, sous certaines conditions, sa confiance. C'est dans ce contexte-là qu'il faut lire et comprendre la théorie de l'État de droit de Slobodan Jovanović. En effet, à la place de la démocratie, entendue comme le gouvernement du peuple par le peuple auquel Jovanović n'a jamais fait confiance, il a développé une théorie selon laquelle ce n'est pas la société civile, mais l'État rationnellement organisé qui assure le meilleur cadre institutionnel pour le plein développement d'une communauté d'hommes libres. Néanmoins, au contraire des auteurs allemands, les auteurs serbes insistaient également sur «l'État» que sur le "droit». Certes, l'idéal allemand d'un État rationnellement organisé les passionnait, mais leur intérêt primordial était de s'en 
servir pour protéger les libertés individuelles devant la menace de la réapparition d'un despotisme nouveau. Alors, si les auteurs serbes, Slobodan Jovanović en tête, affirmaient que l'État de droit est la conception moderne de l'État et du droit, c'est parce qu'ils l'ont conçu comme le meilleur moyen pour un Rule of Law réussi.

En ce sens, l'idéal de l'État de droit s'imposait aux Serbes par la force d'un principe inhérent à chaque État civilisé. Et c'est bien cette universalisation de l'État de droit que dévoile la position de Slobodan Jovanović. En effet, d'après lui, l'État de droit n'est que la description de l'État moderne. Il s'agit de la représentation du modèle étatique qui l'a emporté sur toutes les autres métamorphoses historiques de l'organisation du pouvoir public, c'est-à-dire basé sur la norme juridique comme moyen principal de son organisation, de son fonctionnement et de son autolimitation rationnelle. Imposé comme forme d'organisation étatique la plus répandue, l'État de droit est intégré dans les fondements constitutionnels de tous les pays occidentaux ou en voie d'occidentalisation. Pour Jovanović, il ne semble pas exister d'alternative.

On trouve une belle illustration de cette théorie chimérique d'un État fort en tant que protection la plus efficace de la liberté individuelle chez Živojin Perić. D’après lui,

L'État présente une dimension supérieure, construite d'une manière consciente et rationnelle contre les masses inorganisées et les forces sociales dépourvues de la conscience. L'ordre, conscient et rationnel, n'est pas un mot vide. Le rationalisme auquel il pense est le rationalisme qui coïncide l'unicité dans l'action et dans la conduite qui possède sa logique immanente et ses lois. L'État, en tant qu'être rationnel, est soumis à la même rationalité. (Tasić, 1938, 18).

L'autorité d'un État provient, donc, de sa rationalité présupposée. Il est fort, car rationnel. D'un autre côté, Perić accepte également le vieil axiome libéral de la nécessité d'une démarcation claire entre vie privée et vie publique, en définissant seulement la seconde comme le domaine du droit. En tant que réglementation normative de l'État, le droit ne porte donc pas sur toutes les activités humaines, mais uniquement sur les questions d'organisation sociale. Autrement dit,

Si toutes les normes sociales quel que soit leur contenu étaient des normes de droit, cela signifierait que nous serions en présence d'un Coran qui règle toutes les manifestations de la vie humaine à commencer par le manger et le boire jusqu'à la vie politique: il n'y a pas dans pareilles législations [...] deux domaines de vie: domaine privé et domaine public, tout était vie publique ou, si l'on veut, tout était vie privée. (Péritch, 1935, 231).

51 Cependant, le discours libéral de Perić est également controversé, car si selon lui le droit n'est qu'un moyen dont l'usage reste exclusivement réservé au domaine public, l'autonomie de la sphère publique reste ambiguë. En effet, d'après Perić, seul l'État est compétent pour déterminer précisément la trajectoire de cette ligne de démarcation. Ainsi, si l'on se demande où se terminent la vie privée et le champ de l'action autonome, il faut s'adresser à l'État et à ses décisions législatives. À vrai dire, bien qu'il soit près d'appeler l'État maître, et même tyran, il ajoute que «L'État n'édicte des lois, c'est-à-dire qu'il ne limite notre liberté et notre activité que dans la mesure où cela est nécessaire pour l'existence de la société et de son évolution » (Idem). Et cette limite qui s'impose à l'État est sa rationalité même, qui doit absolument suivre les idées du progrès. Par conséquent, « à présent ce sont l'idée de liberté et celle de paix qui luttent entre elles pour la prédominance» (id.). Tout comme chez Jovanović, on trouve chez Perić le concept d'État comme ultime arbitre dont la rationalité supérieure est seule capable de résoudre le problème primordial de la théorie politique : trouver le juste 
équilibre entre la liberté individuelle et la paix sociale assurée pour tous ${ }^{16}$. On est très loin de la démocratie libérale et de son concept d'État au service d'une société civile autonome. Hors d'État nul la liberté. Littéralement.

\section{Légitimité de l'État - la volonté générale vs la volonté du peuple} sa conception de l'État, dans le sens où il ne suit que les idéaux connus du rationalisme moderne. Adepte de Rousseau, qui savait reconnaître que le vrai problème de la politique réside dans la réconciliation de la liberté individuelle et de l'intérêt général (id., 50), Jovanović, à l'instar du Genevois, reconnaît le rôle de médiateur de l'organe législatif dont le produit, la loi, est la juste mesure des deux revendications mentionnées. Et bien que la conception de l'Assemblée nationale proposée par le Serbe ne reflète pas la composition de celle de Rousseau, leur foi dans sa capacité délibérative reste immense. Cependant, tandis que le second voit dans l'Assemblée nationale le représentant du peuple en tant que titulaire exclusif de la souveraineté et donc l'unique corps apte à exprimer la volonté générale, le premier, méfiant par rapport au peuple, n'y voit qu'un membre d'une entité bien plus large et importante - l'État même en tant que véritable porteur de la rationalité politique.

56 En ce sens, il est symptomatique que dans le discours de Jovanović ce soit toujours l'État, catégorie quasi métaphysique, et non le législateur, qui est désigné comme l'entité politique apte à découvrir et à réaliser l'intérêt général. À l'instar d'une longue lignée de penseurs politiques allemands nés dans l'autocratie prussienne et trouvant leur apothéose ultérieure dans la philosophie de Hegel, Jovanović voit dans l'État et ses organes l'unique source véritable d'une politique rationnelle, responsable et 
objectivement menée. Dans ces conditions, ce n'est pas Assemblée nationale en tant que corps démocratiquement élu par le peuple qui assure l'émanation correcte de la volonté générale, mais bien l'Assemblée nationale comme organe d'état dont le caractère étatique, non démocratique, assure l'enquête effective pour l'intérêt commun. Selon Jovanović, ce sont les organes étatiques qui sont appelés à exprimer l'intérêt abstrait et général, ou ce qui reste la même chose, mais illustre mieux l'essence de sa pensée, l'intérêt étatique. C'est parce qu'ils sont au service exclusif de l'État. En effet, il est entièrement persuadé que ces organes et leurs agents peuvent se mettre autant audessus des intérêts particuliers de son groupe que de leurs intérêts propres.

Ainsi, en dernière analyse, l'idéal de l'État de droit en tant qu'État neutre s'appuie sur la qualité de ses agents - les êtres humains à son service. Il nous semble qu'ici, les vieux idéaux de la théorie politique allemande obtiennent leur parfaite incarnation serbe : c'est la bureaucratie professionnelle que Slobodan Jovanović reconnaît comme la meilleure garantie de la neutralité de l'État. Il considérait les agents publics, dépourvus d'autre vocation professionnelle, comme les serviteurs loyaux de l'État. Selon lui, « le pouvoir étatique est capable d'être neutre parce qu'il est bureaucratisé. Ou, plus simplement : c'est le bureaucratisme qui garantit la neutralité » (Basta, 1998, 352).

Il faut remarquer qu'il soulignait que, dans l'État moderne, le pouvoir judiciaire et le pouvoir exécutif sont dans une bonne mesure bureaucratisés. Par conséquent, le degré de neutralité acquis par leur travail est forcement élevé, et l'intérêt général particulièrement bien protégé. En revanche, étant donné que le pouvoir législatif, comme l'unique branche du pouvoir public apte à vouloir pour l'État, n'était pas bureaucratisé, elle s'imposait à Jovanović comme le plus grand problème. Comment garantir la neutralité de la loi, si on laisse la composition de l'Assemblée nationale aux aléas des jeux politiques et pire encore, aux choix des masses populaires? À part l'élitisme, c'est-à-dire l'incorporation de la couronne et du sénat dans le procédé de confection de la loi, Jovanović trouve encore une solution à ce problème - la prépondérance du gouvernement, en fait le pouvoir exécutif sur le parlement: le modèle constitutionnel qu'il croyait trouver dans le système britannique.

Une lecture plus soigneuse de cette partie de son œuvre consacrée à la crise de l'État de droit, dans la période suivant la Première Guerre mondiale, montre sa réticence envers le modèle constitutionnel de la ${ }{ }^{e}{ }^{e}$ République et ses sympathies mal cachées pour les institutions politiques de Grande-Bretagne. D'ailleurs, c'est exactement la riche expérience du constitutionnalisme anglais qui lui a fourni des arguments en faveur d'un parlement bicaméral et d'un rôle actif réservé à la couronne.

Le reproche principal de Jovanović fait à la Troisième République était la prévalence du parlement sur le gouvernement. Aussi, la faiblesse de la position institutionnelle du gouvernement français devient évidente à cause de la discipline laxiste de sa bureaucratie. En France, les agents publics s'organisent facilement en syndicats et se positionnent par rapport au gouvernement tout comme les ouvriers vis-à-vis des capitalistes. Dans cette position, ils sont parfaitement capables de manipuler l'autorité du gouvernement en vue de réaliser les intérêts de leur propre groupe. Alors, au lieu d'effectivement garantir la protection de l'intérêt commun par sa neutralité politique, la bureaucratie française dénaturée s'est imposée comme une joueuse indépendante et intéressée. Tandis qu'en Angleterre, le gouvernement garde l'autorité sur sa bureaucratie, et même sur la majorité parlementaire, le gouvernement français est 
complètement soumis au parlement et sans contrôle réel sur son propre appareil administratif, voire, parfois, même dépendant de lui (Jovanović, 1991a, 431).

En effet, la critique que faisait Jovanović au système parlementaire serbe constitué en 1903 portait exactement sur la tendance à l'accroissement du pouvoir du parlement vis-à-vis du gouvernement. La réduction du rôle de ce dernier était la preuve « qu'on a tout de suite commencé à exagérer avec l'application pratique du régime parlementaire introduit par la nouvelle Constitution » (Jovanović, 1932a, 49). Ainsi, selon Jovanović, dans le nouveau régime, le concept du ministre politicien l'a emporté sur celui du ministre expert issu du milieu bureaucratique, car désormais « on choisit les ministres parmi les députés. Alors, tandis que les ministres d'autrefois étaient recrutés au Conseil en tant que chancellerie principale d'État, aujourd'hui on les recrute à l'Assemblée " (idem). Encore une fois, les sympathies de Jovanović sont du côté des bureaucrates instruits, et non du suffrage universel.

\section{Validité du droit - la sanction étatique vs l'adoption individuelle}

En suivant les préceptes de la théorie allemande du droit étatique, Jovanović devait tôt ou tard arriver à la doctrine normativiste sur la validité d'une norme juridique. D'après lui, la notion de la validité au sens juridique ${ }^{17}$ se rapport à la norme dont l'application est exigée par l'État. Autrement dit, c'est l'État qui prend en charge l'application à venir de cette norme. Cependant, il faut remarquer que valide est la norme dont l'application est exigée ou assurée par l'État, mais dont l'application elle-même n'est pas la cause de sa validité. C'est la garantie étatique de l'application, et non l'application en soi, qui compte. La validité est plutôt l'applicabilité de la norme que son application. Ou bien, pour citer Jovanović, la norme juridique, c'est-à-dire celle adoptée par l'État, jouit de cette validité en tout cas (id.).

C'est pourquoi l'énoncé que la norme juridique est valide n'est qu'une tautologie, car d'après Jovanović les deux adjectifs signifient la même chose - que la norme est étatique. Et ce caractère étatiste de la norme juridique et valide provient, d'abord, de la sanction étatique. Le fait qu'une norme soit valide ne signifie pas autre chose que l'État a décidé de protéger son application par son monopole de la contrainte physique, à la limite, en sanctionnant sa transgression. Ainsi, on voit que dans la théorie étatique du droit, la notion de sanction est d'une importance cruciale. « Le droit est le droit et il est en vigueur parce que ses normes ont été prescrites par l'État et parce que leur application, en cas échéant, l'État l'assure par sa sanction » (Basta, 1998, 349) Le rôle de la sanction reste crucial pour les autres auteurs serbes. Ainsi, Živojin Perić écrit que "C'est donc le législateur qui, faisant la fonction du souverain (alors, de l'État), est la source unique des droits, car il n'y a que lui qui peut sanctionner les règles de droit, par la sanction sans laquelle ces règles resteraient à l'état platonique, théorique, et n'auraient plus de force que les règles non juridiques, par exemple, les règles de la morale ou les conventions sociales... » (Péritch, 1935, 231).

À notre avis, de cette notion de "validité au sens juridique » découlent au moins deux conséquences qui rapprochent considérablement la théorie du droit étatique d'une sorte de normativisme « dure ", c'est-à-dire qui définie la validité en tant qu'existence spécifique de la norme, différente de l'existence empirique, et qui y rattache son caractère obligatoire. Pourtant, ce ne sera pas le normativisme de Jovanović en tant que tel, mais plutôt son effet politique, qui nous intéressera ici. 

insistent sur la prétendue «obéissance autonome au droit » selon lesquelles le droit oblige seulement s'il est préalablement adopté intimement par le sujet de l'obligation normative. La validité morale, c'est-à-dire l'adoption autonome d'une norme par ses destinataires, est donc sans signification. Au contraire, d'après Jovanović, au sens juridique, la loi oblige tout le monde, même celui qui ne connaît pas son contenu concret. Les convictions personnelles et les motifs des particuliers sont simplement sans importance. En fait, il dit que

... la force obligatoire de la loi se manifeste le plus amplement là où l'individu essaye de se conduire contre la loi. Bien que dans ce cas l'individu montre d'une manière très claire qu'il ne possède aucune conviction intérieure qui pourrait lui faire respecter la loi, l'État le force à respecter la loi ; sa pression extérieure vient à la place des motifs intérieurs dont l'individu manque. (id.,59)

Il pourrait sembler que cette position de Jovanović n'est pas spécifique. Néanmoins la spécificité de cette position devient évidente si on la replace dans le contexte de sa pensée $a$-démocratique dans son ensemble. Ainsi, si on accepte que l'opinion du destinataire ultime d'une norme juridique ne joue aucun rôle dans sa validité, on est en droit de se demander si le législateur devrait prendre en considération cette opinion à l'occasion de la délibération qui précède la production de la norme. D'ailleurs, chez Jovanović, la mission du législateur est de dévoiler la volonté générale, qui, on le sait, ne coïncide pas avec la volonté de tous ni, a fortiori, de certains. 
71 À la fin de l'analyse, c'est l'État, et seulement lui, qui s'impose comme le dernier arbitre sur l'acceptabilité d'une règle en tant que norme juridique. En ce sens, la validité juridique de Jovanović fondée sur un jugement politique de l'État et la confiance en sa rationalité quasi divine devient l'unique source de la légitimité du droit et la meilleure justification pour l'appellation de sa théorie comme celle du droit étatique.

\section{Conclusion}

72 À première vue, la dimension libérale de la théorie politique et juridique serbe est indéniable, car, face au despotisme des princes, elle propose l'État de droit qui se fonderait sur le règne de lois, le respect des droits individuels et la séparation des pouvoirs autant que sur les autres mécanismes classiques du constitutionnalisme libéral moderne. Cependant, le rejet de la souveraineté populaire, l'affirmation des éléments élitistes, l'omniprésence de l'État et, analogiquement, le manque d'engouement pour l'autorégulation de la société dominent dans ce discours et témoignent en faveur d'une conclusion plus austère. En effet, si le profil de cet État de droit à la serbe ressemble au modèle anglo-saxon, c'est son de face qui nous révèle ces profonds traits «prussiens ». Tout comme son véritable modèle germanique, l'État de droit des théoriciens serbes avec Slobodan Jovanović en tête, n'a jamais été conçu comme le service de ses citoyens, mais comme une entité abstraite, hyper rationnelle, qui gouverne la société, d'une manière ou d'une autre, faible ou retardée.

En réalité, l'idée d'une société civile qui englobe l'État, et dont ce dernier n'est qu'un serviteur, est connue, mais peu convaincante et mal reçue parmi les Serbes. Les derniers se sont rendu compte que la société serbe du début $d u x^{e}$ siècle était profondément traditionaliste, patriarcale et pauvre en institutions civiles bien établies. C'est pourquoi, bien que toujours intéressés par la progression des droits et libertés individuels, ils sont rarement libéraux au sens classique du mot, et leur libéralisme est d'une certaine manière « étatique ». À savoir que leur programme libéral a besoin d'un État fort qui va, par ses mesures politiques et la loi comme son arme civilisatrice, présupposer la société des individus libres et autonomes. C'est à lui, en tant qu'organisme rationnellement structuré, d'accomplir la mission culturelle et d'organiser une société civile moderne dans une Serbie terriblement sous-développée. Ainsi, ce n'est pas la société civile qui engendre l'état, mais l'État qui engendre la société civile.

Le paradigme serbe est ainsi bien opposé à celui du libéralisme anglo-américain. Les libéraux serbes expriment leur peur envers les idées de la démocratie basée sur la souveraineté du peuple dans sa forme mythique - le gouvernement du peuple, par le peuple et pour le peuple. Ils ne lui font pas confiance, et s'ils sont toujours favorables au gouvernement pour le peuple, ils ne le conçoivent jamais comme celui du peuple, et surtout pas comme celui par le peuple. En outre, ils préconisent des mécanismes institutionnels aptes à bloquer, le cas échéant, la possibilité d'une prépondérance politique des masses populaires et de leurs représentants. D'où l'idée du bicaméralisme et du rôle prononcé de la couronne dans la législation. D'où, enfin, l'idée du droit en tant que produit rationnel d'un État mené, non par le peuple, mais toujours en faveur du peuple mal instruit. C'est la face « élitiste » du libéralisme serbe.

Et pourtant, cette heureuse union entre étatisme et élitisme dans la pensée libérale serbe n'étonne pas tellement si l'on prend en considération l'origine de l'élite sociale 
dans la Serbie de l'époque. Dans ce pays dépourvu d'une bourgeoisie puissante et d'une classe moyenne établie, le seul promoteur des idées libérales était l'intelligence, dont la majorité faisait partie de la bureaucratie étatique. Une telle intelligence était complètement dépendante de l'État, qui manipulait effectivement la mobilité sociale de ses agents. Comme c'est déjà bien remarqué par Dubravka Stojanović (Stojanović, 2005, 129), c'est l'État qui les a scolarisés, en assurant les bourses aux universités étrangères, puis leur a attribué les postes et fonctions, les salaires et carrières, le statut social privilégié, enfin la prospérité professionnelle et la dignité personnelle. La couche sociale composée des citoyens les mieux formés était donc liée à l'État par un contrat spécial. C'est l'État qui l'a créé, non l'inverse. Normalement, dans ces conditions, on n'aurait pas pu s'attendre à ce que cette intelligence s'impose comme l'adversaire de l'État et de son interférence dans le domaine de la société civile. Bien au contraire, elle était le plus grand partisan de cette intervention étatique. Sans autre connaissance, isolée au large d'une mer agraire, sceptique vis-à-vis de l'initiative privée, de l'association et du libre marché, cette élite serbe a reconnu dans l'État l'unique force rationnelle capable d'assurer le progrès national. Toutefois, l'État ne serait capable d'accomplir sa tâche libératrice que si les éléments rationnels prévalent dans son organisation. En ce sens, s'il est imaginé comme une démocratie, l'État de droit des théoriciens serbes n'est qu'une démocratie étroite ou élitiste. Ainsi, à côté des masses populaires et de leurs représentants parlementaires, il faudrait juxtaposer les éléments aristocratiques et technocratiques comme les mécanismes correctionnels et une sorte de contrepoids politique. Ce concept de l'État ne se fonde pas tant sur la foi dans le peuple et ses capacités démocratiques, que dans celles des individus exceptionnels. On est même tenté de conclure que les libertés individuelles ne sont ici que les conditions préalables au plein épanouissement des individus prédestinés à gouverner les masses. En fin de compte, ils prônent un système politique dans lequel l'élite sociale promeut les libertés individuelles sur lesquelles se base sa propre autoreproduction ${ }^{18}$.

Forcement, cette interprétation de l'État de droit à la serbe, met directement en cause la représentation classique de l'œuvre de Slobodan Jovanović et de ses contemporains en tant qu'une théorie juridique et politique libérale dans le sens moderne. De cette manière, notre analyse rend sa modeste contribution à la déconstruction du mythe national de «l'âge d'or de la démocratie serbe ».

À l'origine, c'est un récit d'origine très confortable : à partir du fait historique que le régime parlementaire instauré en 1903 est issu d'un dur combat contre l'absolutisme des princes serbes, mené résolument depuis la libération en 1804, puis tout au long du $\mathrm{XIX}^{\mathrm{e}}$ siècle, le mythe rappelle que, chez les Serbes, les deux processus historiques - celui de la libération nationale et celui de la lutte démocratique - ont coulé parallèlement pour culminer de concert au début du $\mathrm{xx}^{\mathrm{e}}$ siècle, par le triomphe dans les guerres libératrices (1912-1918), gagnées par le Royaume de Serbie, une monarchie constitutionnelle et parlementaire. Riche de ses fonctions, ce mythe a d'abord servi de «preuve» de la supériorité civilisatrice des Serbes sur les autres peuples yougoslaves et, par conséquent, de prétexte à leur hégémonie politique : les Serbes n'étaient pas seulement la seule nation parmi les Slaves du sud à réussir à gagner son indépendance, mais aussi les seuls capables de reconnaître et d'adopter les valeurs libérales et démocratiques. Ensuite, au début des années 1990, il "témoigne» en faveur de l'incompatibilité entre l'esprit serbe et le communisme, ce dernier, du reste, imposé par un Croate, Tito. Enfin, aujourd'hui, ce même mythe sert de prétexte au ralentissement des réformes urgentes. D'après cette image mythologique, «le passé serbe a coulé 
parallèlement à celui européen, en traversant les mêmes phases, et, du coup, le transfert des concepts européens n'était pas un procès douloureux qui, pour avoir du succès, devrait confronter les obstacles nombreux » (Stojanović, 2003, 129). Autrement dit, on avait déjà eu une fois la démocratie et, bien sûr, on l'aurait de nouveau. En définitive, on se retrouve enfermé dans l'imaginaire dangereux du mythe de l'excellence nationale, inséparable de la notion de collectivité restreinte et du rétrécissement de l'espace social autour de petits groupes communautaires, solidairement refermés sur eux-mêmes (Girardet, 1986, 181).

C'est le mérite des travaux de l'historiographie critique serbe, qui a finalement dévoilé toute une série d'erreurs concernant cette interprétation nationale romantique. Il s'agissait d'une démarche dure, peu populaire et politiquement risquée, de la démythification comme une entreprise introspective tant nécessaire aux nations balkaniques et au progrès de leur émancipation tardive. Ces dernières, "n'ont pas encore appris à distinguer le passé et le présent, et c'est pourquoi elles ne sont pas aptes à atteindre cette objectivité indispensable pour l'interprétation du passé, sans laquelle la conscience historique se libère difficilement et lentement de son aspect mythique en faveur de celui historique proprement dit. Une représentation brouillée de sa propre histoire crée une représentation brouillée de son propre présent, et c'est la raison pour laquelle la conscience sociale et politique des peuples balkaniques est encore aujourd'hui, au seuil du XxI ${ }^{\mathrm{e}}$ siècle, essentiellement déterminée par les erreurs, fictions et mythes » (Popović-Obradović, 2008, 51).

Les résultats de cette " déconstruction du mythe », « du long chemin de la démocratie serbe " proposent une image moins héroïque et, surtout, plus logique de l'histoire nationale. D'après cette réinterprétation, l'origine du mythe réside dans une confusion des concepts - plus précisément, dans l'assimilation simplificatrice de la démocratie à l'égalité. À partir d'une idée primitive de la démocratie comme société dépourvue de hiérarchie, certains intellectuels serbes de l'époque ont reconnu leur pays, ce merveilleux "paradis des pauvres », comme le terrain autochtone et le champion de la démocratie. À leurs yeux, la Serbie archaïque a déjà réussi à réaliser l'idéal que les autres pays européens essaient toujours d'atteindre : l'égalité parfaite de tous. Pleins d'orgueil national, ils se vantaient de cette démocratie serbe en tant que situation authentique, ou résultat spontané de l'esprit national. Naturellement, toutes ces voies de développement, venues de l'Occident, qui menaient vers la stratification sociale ont été considérées comme une menace directe à la préservation de cette prouesse du peuple serbe.

80 Par notre étude, on a essayé de relativiser une représentation bien ancrée qui échappe à la démarche de l'historiographie critique serbe et selon laquelle la théorie dominante du droit et de l'État serbe de l'époque qui coïncide avec « l'âge d'or de la démocratie serbe ", suivait fidèlement la ligne droite de la pensée moderne et, par conséquent, était le seul véritable partisan du libéralisme politique au sens moderne. Ainsi, si le discours et la pratique politique serbe du début $d u x^{e}$ siècle se sont faussement présentés comme démocratiques au sens moderne, reste que, du moins, la pensée théorique de la même époque était fidèle aux idéaux de la société et de l'État libéral. Malgré tout, la bannière de la démocratie libérale aurait en Serbie ses porteurs. l'hypothèse que le projet de démythification a déjà bien avancé au niveau de l'origine idéologique (Perović, 2006), de la culture politique (Stojanović, 2003) et du cadre 
institutionnel (Popović-Obradović, 2008) de cette démocratie douteuse à la serbe, mais qu'il a contourné la production théorique en laissant intouchable le dogme sur la prétendue modernité libérale et démocratique de la théorie de droit et de l'État serbe au tournant du siècle.

\section{BIBLIOGRAPHY}

Bartoš, Milan, (1954), «In memoriam Živojin Perić», Anali Pravnog fakulteta u Beogradu, nº 1, p. 118-119.

Basta, Danilo, (1998), «Slobodan Jovanović kao teoretičar države i prava», in M. Jovičić (dir), Slobodan Jovanović, ličnost $i$ delo, Belgrad: Srpska akademija nauka i umetnosti, p. 337-366.

Basta, Danilo, (2001) «Slobodan Jovanović i Hans Kelzen», Anali Pravnog fakulteta u Beogradu, $\mathrm{n}^{\circ} 1-4$, p. 25-43.

Bataković, Dusan, (1997), «Francuski uticaji u Srbiji 1835-1914, četiri generacije parizlija», Zbornik Matice Srpske za istoriju, $\mathrm{n}^{\circ}$ 56, p. 73-95.

Čekić, Milos, (1908), «Kulturni smisao jedne stranke», Nedeljni pregled, ${ }^{\circ}$ 4, p. 72-78.

Đorđević, Dimitrie, (1985), «Srpsko društvo 1903-1904», Marksistička misao, nº 4, p. 125-136.

Girardet, Raoul, (1986), Mythes et mythologies politiques, Paris : Éditions du Seuil.

Jovanović, Slobodan, (1897), «O suverenosti», Branič, nº 4, p. 69-73; 85-88; 107-110.

Jovanović, Slobodan, (1932a), «Naše ustavno pitanje u devetnaestom veku», in S. Jovanović. Političke i pravne rasprave I, Belgrad: Geca Kon, p. 1-58.

Jovanović, Slobodan, (1932b), «Dvodomni sistem», in S. Jovanović, Političke i pravne rasprave II, Belgrad: Geca Kon, p. 1-78.

Jovanović, Slobodan, (1938, «Perić o vladalačkoj vlasti», Arhiv za pravne i društvene nauke, $\mathrm{n}^{\circ}$ 1-2, p. 6-10.

Jovanović, Slobodan, (1991a), Država, Belgrad: BIGZ.

Jovanović, Slobodan, (1991b), «O totalitarizmu», in S. Jovanović, Iz istorije i književnosti. Belgrad: BIGZ, p. 141-174.

Jovanović, Slobodan, (1991c), «O dvodomnom sistemu», in S. Jovanović, Sabrana dela-Političke i pravne rasprave I-III. BIGZ: Belgrad, p. 233-271.

Jovanović, Slobodan, (1991d), Iz istorije političkih doktrina, Belgrad: BIGZ.

Khun, Thomas, (1962), The Structure of Scientific Revolutions, Chicago: University Chicago Press.

Ković, Milos, (2010), « La Révolution française et l'élite serbe (1889-1935) », in Bataković Dusan T. (dir), La Serbie et la France : une alliance atypique, Institut des Études balkaniques, Belgrade : Académie serbe des Sciences et des Arts, p. 187-205.

Leboyer, Olivia, (2012), Élite et libéralisme, Paris : CNRS éditions. 
Milovanović, Milovan, (1997), «Jedan ili dva doma», in M. Mlovanović. Državno pravo i druge ustavno-pravne studije, Belgrad: Službeni glasnik, p 269-287.

Novaković, Stojana, (1908), «Umesto predgovora», in Ž. Perić, Političke studije, Belgrad: Geca Kon, p. 5-13.

Pavković, Alexandar, (1998), «Čemu liberalizam? Razmatranja Slobodana Jovanovića», in M. Jovičić (dir), Slobodan Jovanović, ličnost i delo, Belgrad: Srpska akademija nauka i umetnosti, p. 673-678.

Perić, Živojin, (sous le pseudonyme de Lannes), (1910), «Demokratizam sa nacionalnog gledišta», Nedeljni pregled, $\mathrm{n}^{\circ} 12$, p. 180-184.

Perić, Živojin, (1908), Političke studije, Belgrad: Geca Kon.

Péritch, Jivoïn, (1903), La nouvelle constitution serbe, Paris : Impr. Général Lahure.

Péritch, Jivoïn, (1904), Le monarque constitutionnel, Paris : Albert Fontemoing.

Péritch, Jivoïn, (1935), « Quelques observations sur le problème des sources du droit et la fonction de la loi ", in Les sources du droit, Recueil d'études en l'honneur de François Gény, t. II, Paris : Recueil Sirey, p. 227-242.

Perović, Latinka, (2006), Između anarhije i autokratije. Belgrad: Helsinški odbor.

Petrović, Milan, (1998), «Pravo u teoriji države Slobodana Jovanovića», in M. Jovičić (dir), Slobodan Jovanović, ličnost $i$ delo, Belgrad: Srpska akademija nauka i umetnosti, p. 383-398.

Popović-Obradović, Olga, (1998), «"Parlamentarna hronika“ Slobodana Jovanovića», in M. Jovičić (dir), Slobodan Jovanović, ličnost $i$ delo, Belgrad: Srpska akademija nauka i umetnosti, p. 569-582. Popović-Obradović, Olga, (2008), Kakva ili kolika država, Belgrad: Helsinški odbor.

Protić, Milan St., (1990), Radikali u Srbiji, ideje i pokret, 1881-190, Belgrad: Balkanološki institut SANU.

Protić, Milan St., (1998), «Slobodan Jovanović - konzervativac ili demokrat», in M. Jovičić (dir), Slobodan Jovanović, ličnost $i$ delo, Belgrad: Srpska akademija nauka i umetnosti, p. 663-672.

Spasojević, Živan, (1989), Nacrt jedne opšte teorije prava, Belgrad: Srpska akademija nauka i umetnosti.

Stanovčić, Vogislav, (1998), «Apsolutna vladarska vlast, birokratija, demokratija i ustavna vlada (u delima Slobodana Jovanovića)», in M. Jovičić (dir), Slobodan Jovanović, ličnost i delo, Belgrad: Srpska akademija nauka i umetnosti, p. 619-652.

Stojanović, Dubravka, (2003), Srbija i demokratije 1903-1914, Belgrad: Udruženje za društvenu istoriju.

Stojanović, Dubravka, (2005), «Ulje na vodi, politika i društvo u modernoj istoriji Srbije», in Lj Dimić, D. Stojanović Dubravka, Jovanović Miroslav (dir), Moderna Srbija 1804-2004, Belgrad: Udruženje za društvenu istoriju, p. 115-148.

Sundhaussen, Holm, (dir) (1989), Historische Statistik Serbiens 1834-1914, Munich: mit europäischen Vergleichsdaten.

Tasić, Tomislav, (1938), «Profesor Živojin Perić kao pravni filozof», Arhiv za pravne i društvene nauke, $\mathrm{n}^{\circ} 1-2$, p. 16-24

Troper, Michel, (1994), Pour une théorie juridique de l'État, Paris : PUF. 
Zimmermann, Werner G., (1962), Valtazar Bogišić, 1834-1908, Ein Beitrag zur sudslawischen Geistes und Rechtsgeschichte im 19 Jahrhundert, Wiesbaden: Franz Steiner Verlag.

Živanović, Toma, (1959), Sistem sintetičke pravne filozofije, Belgrad:Naučno delo.

\section{NOTES}

1. Entre 1937 et 1941 Jovanović était président du Club culturel serbe qui a réuni les intellectuels de droite. À la veille de la guerre, dans une situation politiquement difficile, il accepte l'engagement politique et devient vice-président du gouvernement yougoslave. Après la défaite militaire en 1941, il a quitté le pays avec le roi et le gouvernement dont il a été le président en exil entre janvier 1942 et juin 1943. À cause de cet engagement politique et de sa réputation nationaliste, le nouvel État communiste l'a condamné à vingt ans de prison, ainsi qu'à la perte de ses droits politiques et civils, la confiscation de ses biens et la perte de sa citoyenneté. Il est mort à Londres, pauvre et apatride. Après la chute du régime de Milošević, Slobodan Jovanović a été réhabilité en 2007 par le tribunal de Belgrade, et ses ossements ont été exhumés, transférés et enterrés en Serbie en décembre 2011. Aujourd'hui, c'est probablement ce mélange du national et du démocratique chez Slobodan Jovanović qui en fait l'un des "gourous» de la droite serbe, des partis politiques et organisations proches de l'Église orthodoxe serbe et de la tradition populaire, et réservés à l'égard du libéralisme occidental.

2. Ce que Slobodan Jovanović était pour le droit public serbe, Živojin Perić l'était pour le droit civil - une autorité incontestable. Mais, bien que civiliste de réputation internationale (professeur à l'Académie de droit international de La Haye, Doctor Honoris Causa de l'Université de Lyon en 1933), Perić s'intéressait également à la théorie politique et au droit public. Au contraire de Slobodan Jovanović, Perić était très engagé en tant que membre, idéologue et député du Parti du progrès. D'une personnalité forte, il s'est profilé comme l'intellectuel indépendant, presque dissident. Ainsi, avant 1914, il est connu par son attitude conservatrice et son austrophilie dans un milieu très russophile comme l'était la Serbie de cette époque. Il garde la même réputation entre les deux guerres mondiales quand il est partisan du fédéralisme yougoslave et plaide pour le compromis avec les Croates. Pendant la Seconde Guerre mondiale, il sympathise avec le projet de Nouvelle Europe (Bartoš, 1954,118) et participe en tant qu'expert juridique au gouvernement collaborationniste du général Milan Nedić (Popović Obradović, 2008, p. $300 \mathrm{~s})$.

3. Bien sûr, la seule existence d'un paradigme ne signifie pas l'absence d'approches alternatives, telles que, par exemple, l'idéologie radicale représentée dans l'œuvre de Gligorije Geršić, le socialisme de Jovan Žujović et de Jovan Skrerlić, ou une philosophie synthétique du droit de Toma Živanović. Cependant, dans l'Université de Belgrade, c'est la doctrine de l'État de droit qui a régné souverainement jusqu'en 1945.

4. Slobodan Jovanović explique ce concept brièvement de la façon suivante : « La souveraineté est un pouvoir - le pouvoir d'exprimer une volonté qui impose l'obligation à tous les membres d'une société politique [...] il doit entièrement appartenir à une seule autorité. C'est exclusivement l'autorité du législateur qui dispose du pouvoir de la création de la volonté suprême » (Jovanović, 1897, 108).

5. Et c'est une thèse "paradigmatique ", c'est-à-dire très répandue et bien établie parmi les théoriciens serbes, dont témoigne particulièrement un passage chez Toma Živanović « En réalité, le droit et l'État [...] dans leur existence dépendent l'un de l'autre dans le sens que la genèse du premier conditionne la genèse du second et inversement, et, par conséquent, ils représentent par leurs genèses respectives, deux entités logiquement concomitantes ", (Živanović, 1959, p. 101). 
6. Ce que Kelsen explique dans son essai Gott und Staat de manière très illustrative.

«En définitive, la théorie traditionnelle est dans sa structure comparable à la religion. Dans les deux cas, on représente l'unité d'un ordre par une hypostase, Dieu dans un cas, l'État dans un autre. Dieu est la personnification d'un système de lois naturelles ou encore le point d'imputation de l'ordre général du monde et de même qu'on conçoit un Dieu transcendant au monde, de même on conçoit un État transcendant au droit. Mais on se heurte aussi dans les deux cas au même problème : comment analyser les relations entre un système et son hypostase. On le résout d'ailleurs de la même façon : dans la théologie le dieu surhumain s'incarne en un dieu humain. Le dieu humain se soumet à l'ordre qu'il a lui-même établi en tant que dieu surhumain. De la même manière, l'État se soumet volontairement au droit qu'il a lui-même créé. Cette comparaison ne vise nullement à ridiculiser la théologie ou la théorie traditionnelle, mais à souligner une identité dans la structure de l'argumentation et à montrer que ces thèses relèvent de la croyance, et qu'elles n'ont rien à voir avec la logique, puisque les contradictions logiques d'un système théologique n'affectent en rien la foi. Il est tout à fait possible de conserver la foi, même si l'on a conscience des contradictions logiques du système théologique. Ces contradictions n'affaiblissent pas la foi. Bien au contraire, dénommées «mystères ", elles en deviennent simplement un objet. L'État apparaît ainsi non pas comme une union d'hommes psychophysiques, mais seulement comme un objet de pensée. Comme la religion, il disparait si l'on n'y croit plus », (Troper, 1994, p. 148).

7. Selon Jovanović la théorie pure du droit de Kelsen n'est que la dernière conséquence de l'évolution du concept de l'état de droit. Car, partant de l'idée de limitation du pouvoir étatique par le droit, on est arrivé « à la prépondérance de la norme en tant que telle sur l'autorité en tant que telle - et aux théories comme celle de Kelsen selon laquelle c'est uniquement la norme, non l'autorité, la notion fondamentale de l'ordre juridique " (Jovanović, 1991d, p. 375). Donc, alors que Kelsen est allé trop loin dans cette surestimation de la norme, Jovanović a su garder la mesure et ne pas négliger l'importance de l'autorité étatique. Ainsi, «le premier a vu l'État de droit exclusivement comme l'État de droit. Le deuxième l'a vu comme l'État de droit, mais aussi comme l'État de droit ». (Basta, 2001, 37).

8. Bien qu'on croise aussi des critiques. Par exemple celle de Živan Spasojević qui trouve que la position de Jovanović est éclectique et précise que «Selon son opinion le droit et l'État sont les facteurs équivalents, les deux également réels, mais on n'y voit pas la sortie ni la solution véritable ", (Spasojević, 1989, 93).

9. L'école historique allemande avait, pour autant, ces adeptes parmi les Serbes et disposait d'une influence manifeste sur la culture nationale juridique durant la deuxième moitié du XIX ${ }^{e}$ siècle. Le principal promoteur de l'école s'incarne à l'Université de Belgrade, en la personne du professeur belgradois du droit civil Andra Đorđević (1854-1914). Néanmoins, la contribution de loin la plus remarquée est celle de Valtazar Bogišić (1834-1908), professeur à l'Université d'Odessa et président de l'Institut international sociologique, l'auteur du premier code civil monténégrin de 1888. Le fruit d'une application soigneuse de la méthodologie de l'école historique - la recherche du droit coutumier et puis, sa transposition dans les dispositions législatives - Code civil de Principauté miniature présente le véritable monument du patrimoine juridique mondiale. Sur cette question (voir : Zimmermann, 1962). En Serbie l'influence de l'école s'affaiblie en continue et depuis le début du $\mathrm{xx}^{\mathrm{e}}$ siècle, on ne trouve plus ses représentants parmi les juristes influents.

10. La participation des paysans dans la population de la Serbie de 1900 était d'environ de $85,9 \%$, et il n'y avait que deux villes de moins de 50000 habitants (Sundhaussen, 1989, p. 1023). Le taux d'analphabétisme était particulièrement élevé et allait jusqu'à 79,7 \% (ibidem). De plus, 54,6 \% des foyers cultivaient moins de 5 hectares de terre (Đorđević, 1985, 125).

11. Dans la littérature on peut croiser des opinions différentes. Ainsi, Miloš Ković écrit « qu'entre 1901 et 1920, Slobodan Jovanović fit quand même un pas vers la gauche » en s'appuyant seulement sur un compte rendu de Jovanović, consacré au livre Mirabeau de Louis Barthou de 1913. On ne 
partage pas cette position. Car, même s'il est vrai que Jovanović écrit que « Mirabeau voulait une Monarchie moderne et démocratique où le Roi ne s'appuie pas sur des hautes classes mais sur le peuple tout entier " il est vrai aussi qu'une seule page avant Jovanović remarque que Mirabeau plaidait pour la Monarchie contre la foule. D’ailleurs, le fait que Jovanović dans cet article, s'exprime contre l'aristocratie ne signifie pas qu'il est contre l'élitisme, notamment intellectuel. (Ković, 2010, 197).

12. Cependant, par cette appellation qui également figure dans le titre de notre étude, on ne veut suggérer aucune parenté prétendue entre les doctrinaires français et la pensée concernée serbe. La dernière thèse a été annoncée par Dušan Bataković (Bataković, 2013, 222, 233, 247), mais elle ne fut jamais analysée en détail et elle requiert toujours d'être vérifiée. Sans doute, la ressemblance entre ces deux courants existe, mais ils perdurent des différences au niveau de leurs contextes sociaux, de leurs méthodologies et de leurs sources respectives qui nous rendent plus prudent dans l'emploi du terme. Ainsi, on le prend sous réserve et si l'on parle du libéralisme doctrinaire serbe, c'est seulement pour mieux accentuer le profil conservateur de cette idéologie par rapport au libéralisme classique.

13. En revanche, les autres conservateurs sont restés fidèles à l'idée de la couronne active ou devenus encore plus radicaux, comme c'était le cas avec Živojin Perić, qui a plaidé pour une position forte du roi, presque absolutiste. Voir : Živojin Perić, Stranke i Parlamentarizam u Srbiji, in Nedeljni pregled du 23 novembre 1908, p. 517.

14. Dans la littérature on rencontre d'autres interprétations, par exemple celle de Dušan Bataković qui trouve l'origine du bicaméralisme serbe chez le théoricien français Édouard Leboulaye et ses Questions constitutionnelles de 1872. Selon Bataković c'était Stojan Novaković, président du parti du progrès, qui a interprété, à l'instar du Français, la chambre haute comme unique barrière efficace contre l'instabilité politique marquée par la suite des révolutions, coups d'État, régimes personnels, etc. (Bataković, 1997, 85). De l'autre côté, il est sûr et certain que les thèses de Laboulaye ont été polémisées par Milovan Milovanović, l'idéologue et l'un des ténors de parti radical, dans son traité critique visant la chambre haute. Voir : (Milovanović, 1997, 271).

15. Bien qu'il ait essayé de prouver que le Sénat ne doit pas être forcement l'écho du féodalisme étant la chambre qui réunit la noblesse, il l'a considéré comme un élément politique de l'origine aristocratique dans le sens que ses membres sont souvent nommés et non élus, et que leur mandat n'est pas temporaire. (Jovanović, 1932b).

16. En expliquant cette approche théorique Đorđe Tasić estime que la contradiction entre l'autorité étatique et la liberté individuelle n'existe qu'en apparence. D'après lui, c'est la notion de la souveraineté du peuple qui les réconcilie, tout en assurant la pertinence du paradigme serbe: «...Une fois adopté dans un État, le principe de la souveraineté du peuple fait, et cela provient de soi-même, que le législateur présente l'unique [l'organe - MB] compétent pour créer le droit et la souveraineté entendue ainsi n'est point contraire aux droits individuels des particuliers, mais, bien au contraire, elle les affirment », (Tasić, 1938, 18).

17. Car, à part la validité au sens juridique, il distingue aussi la validité au sens matériel et la validité au sens moral. Et, alors que la dernière ressemble au sentiment subjectif d'obéissance à la norme, la validité matérielle se base sur l'efficacité de la norme, ou en d'autres termes, une norme est matériellement valide seulement si elle est efficace. (Jovanović, 1990a, 63).

18. Dans ce sens Latinka Perović rappelle que c'étaient les membres du Parti du progrès, les progressistes, - les partisans et promoteurs de ces idées élitistes - qui se sont reconnus euxmêmes comme cette élite prédestinée à mener leur pays sous-développé. (Perović, 2006, 197). 


\section{ABSTRACTS}

This paper tries to question one well established representation which escapes the critical approach of contemporary historiography, the idea that the dominant theory of Law and Serbian state during the so called "golden Age of serbian democracy", followed accurately the straight line of political liberalism in the modern meaning. Precisely, the Serbian Rule of Law at the turn of the century, is not, or not only a "Rule of Law", but first of all, faith in the strong Hegelian State as supposedly neutral and highly rationalized identity. Nevertheless of the State is the only guaranty against the negative impact of irrational factors in politics, whatever their origins, despotism of an absolute prince or of non educated people, it is because its rationality is assured by the elitist components of its Constitution, liberal but no democratic. As it depends on its legal normativism, it depends exclusively on its state origin, whatever the opinion of its addressees or of the concerned population.

Cette étude essaye de remettre en cause une représentation ancrée qui échappe à la démarche critique de l'historiographie contemporaine selon laquelle la théorie dominante du droit et de l'État serbe de l'époque qui coïncide avec « l'âge d'or de la démocratie serbe ", suivait fidèlement la ligne droite du libéralisme politique au sens moderne. Précisément, l'État de droit à la serbe au tournant du siècle, n'est pas, ou pas seulement, un Rule of Law, mais avant tout la foi en l'État fort «hégélien » en tant qu'entité prétendument neutre et hautement rationalisée. Cependant, si l'État est la seule garantie contre l'impact néfaste des facteurs irrationnels dans la politique, quelles que soient leurs origines - despotisme du prince absolu ou du peuple non instruit - c'est parce que sa rationalité est assurée par les éléments élitistes de sa Constitution libérale, mais non démocratique. De même qu'elle est fonction de leur normativisme juridique, selon lequel la validité, y compris le caractère obligatoire, d'une norme juridique, dépend exclusivement de son origine étatique quelle que soit l'opinion de ses destinataires, ou de la population gouvernée.

\section{INDEX}

Geographical index: Serbie

Keywords: Theory of Law and State, Rule of law, Liberalism, Elitism, Serbia, Twentieth century, History of Law, Political history

motsclesmk ТЕОРИЈАТА НА ЗАКОНОТ И ДРЖАВА, ВЛАДЕЕЊЕТО НА ПРАВОТО, ЛИБЕРАЛИЗМОТ, ЕЛИТИЗАМ, СРБИЈА, ДВАЕСЕТТИОТ ВЕК, ИСТОРИЈА НА ЗАКОНОТ, ИСТОРИЈА И ПОЛИТИКА

Mots-clés: théorie du droit et de l'État, état de droit, libéralisme, élitisme motsclestr Hukuk Teorisi ve Devlet, Hukuk, Liberalizmin Kuralı, Elitizm, Sirbistan, Yirminci yüzyıl, Hukuk Tarihi, Tarih ve Siyaset

Subjects: Droit, Histoire du droit, Histoire politique

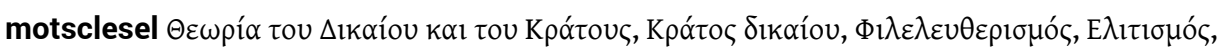

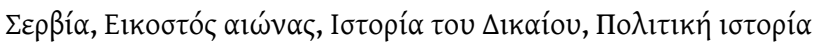

Chronological index: vingtième siècle

\section{AUTHOR}

\section{MARKO BOŽIĆ}

Juriste, docteur de l'Université de Belgrade et de l'Université Paris-Ouest Nanterre 